\title{
AJUSTE SIMULTÂNEO NA PREDIÇÃO DE BIOMASSA AÉREA EM Araucaria angustifolia (Bertol.) Kuntze
}

\author{
Simultaneous adjustment in the prediction of above ground biomass in Araucaria \\ angustifolia (Bertol.) Kuntze
}

\author{
Thiago Wendling Gonçalves de Oliveira ${ }^{1}$, Edberto Moura Lima ${ }^{1}$, Carlos Roberto Sanquetta ${ }^{1}$, Ana \\ Paula Dalla Corte ${ }^{1}$
}

${ }^{1}$ Universidade Federal do Paraná, Programa de Pós-graduação em Engenharia Florestal, Curitiba, Paraná, Brasil - thiagowendling@yahoo.com.br; edberto.moura@gmail.com; carlossanquetta@gmail.com \&anapaulacorte@gmail.com

\begin{abstract}
RESUMO
Atualmente a estimativa da biomassa é feita de forma independente para cada compartimento da árvore, entretanto estudos mostram a importância do ajuste simultâneo para garantir a melhor predição da biomassa por compartimento e total. O estudo teve como objetivo desenvolver modelos alométricos por compartimento e utilizar o ajuste simultâneo de equações para a predição da biomassa da copa, fuste e aérea de Araucaria angustifolia. Foram mensurados diâmetro (dap), altura (ht), densidade básica da madeira ( $\rho$ ) e, pelo método destrutivo, a biomassa verde da copa e fuste. Amostras foram tomadas para a determinação da biomassa seca. Foi realizada correlação de Pearson (5\%) entre as variáveis e testados 10 modelos para a estimativa da biomassa seca dos compartimentos. Os critérios de seleção dos modelos compreenderam $\mathrm{R}^{2} \mathrm{aj}$, Syx\%, AIC, testes de BreuschPagan, Shapiro-Wilk e Durbin-Watson. Após a escolha do melhor modelo foi aplicada a Distância Cook para retirada dos valores outliers. O modelo logaritimizado de dupla entrada (dap e ht) apresentou melhor desempenho para a biomassa da copa e o modelo de tripla entrada (dap, ht e $\rho$ ) para a biomassa do fuste e aérea. A retirada dos valores outliers melhorou as estatísticas de ajuste dos modelos. A aplicação do ajuste simultâneo representou melhora na estimativa da biomassa por garantir a aditividade dos componentes na predição da biomassa total. O uso do dap é importante na estimativa da biomassa do fuste, contudo, deve-se buscar outras variáveis que melhor se relacionem com a biomassa da copa, também podendo melhorar as estimativas com o uso do ajuste simultâneo.
\end{abstract}

PALAVRAS-CHAVE: Alometria, Equações simultâneas, Modelagem florestal.

\begin{abstract}
Nowadays the biomass estimation is done independently for each compartment of the tree, however studies show the importance of the simultaneous adjustment to ensure a better prediction of biomass per compartment and total. The objective of the study was to develop allometric models per compartment and use the simultaneous adjustment of equations for the prediction of tree top, stem and above ground biomass of Araucaria angustifolia. It was measured diameter ( $\mathrm{dbh}$ ), height (ht), wood basic density $(\rho)$ and, by the destructive method, the green biomass of canopy and stem. Samples were taken for determination of dry biomass. Pearson correlation analysis (5\%) was performed between the variables and 10 models were tested for estimation of the dry biomass of compartments. The selection criteria of the models included $\mathrm{R}_{\mathrm{aj}}{ }_{\mathrm{j}}, \mathrm{S}_{\mathrm{yx}} \%$, AIC, Breusch-Pagan, Shapiro-Wilk and Durbin-Watson tests. After choosing the best model, the Cook Distance was applied to remove the outliers values. The logarithmic double input model ( $\mathrm{dbh}$ and $\mathrm{ht}$ ) presented the best performance for canopy biomass and the logarithmic triple entry model ( $\mathrm{dbh}$, ht and $\rho$ ) for the stem and above-ground biomass. The removal of the outliers values improved the adjustment statistics of the models. The application of the simultaneous adjustment represented an improvement in the biomass estimation by guaranteeing the additivity of the components in the prediction of the total biomass. The use of dbh is important in the estimation of the stem biomass, however, it is necessary to look for other variables that best relate to the canopy biomass, also being able to improve the estimates by using the simultaneous adjustment.
\end{abstract}

KEYWORDS: Allometry, Simultaneous equations, Forest modeling. 


\section{INTRODUÇÃO}

Atualmente, as mudanças climáticas representam um dos maiores desafios para a humanidade (IPCC, 2014). Nesse contexto, as florestas exercem um papel importante no ciclo de carbono, onde a estocagem e sequestro do mesmo estão relacionados com as espécies, manejo dos plantios e a sucessão em florestas nativas (WATZLAWICK et al., 2011; SOUZA et al., 2012).

Nesse cenário, a importância de avaliar o estoque de biomassa, através de variáveis de fácil mensuração tornam-se fundamentais para entender o comportamento de estoque do carbono com o crescimento dos indivíduos (SANQUETTA et al., 2003).

A mensuração da biomassa tem assumido importante papel nos inventários florestais, visando a exploração comercial da madeira e do ciclo global de carbono na floresta, pois geralmente assumisse que $50 \%$ da biomassa acima do solo corresponde ao carbono estocado (BASUKI et al., 2009).

A estimativa da biomassa das árvores pode ser feita pela utilização de modelos alométricos, que empregam variáveis de fácil mensuração, como o diâmetro a 1,3 metros de altura e a altura total, para estimar variáveis que apresentam maior dificuldade de mensuração, como a biomassa (SANQUETTA et al., 2014; NICOLETTI, SILVA \& FLORIANI, 2015).

No ajuste dos modelos alométricos geralmente procede-se de forma independente para cada compartimento da árvore (CUBAS et al., 2016; NICODEMO et al., 2016), entretanto, estudos tem mostrado a importância do ajuste simultâneo na estimativa da biomassa total, de modo a garantir a qualidade da estimativa da biomassa por compartimento (BEHLING et al., 2012; VIANA, MARQUES \& FONSECA, 2013).

$\mathrm{O}$ ajuste simultâneo de equações alométricas permite maior precisão na estimativa da biomassa total das árvores do que 0 ajuste independente por compartimento, pois garante a aditividade dos componentes do modelo, visto que a soma das biomassas estimadas por compartimento não assemelha-se a soma das biomassas reais por compartimento, razões pelas quais esse método deve ser difundido e usado nos modelos alométricos (CARVALHO \& PARRESOL, 2003; ANTÓNIO et al., 2007; SANQUETTA et al., 2015).

O objetivo desse estudo foi comparar o ajuste de modelos alométricos individuais para estimativa da biomassa por compartimento e a avaliar o ajuste simultâneo para a estimativa integrada da biomassa por compartimento e aérea em Araucaria angustifolia .

\section{MATERIAL E MÉTODOS}

\section{Caracterização da área de estudo}

Os dados utilizados foram obtidos de um plantio de Araucaria angustifolia, localizado nos municípios de

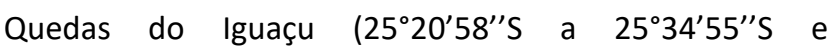
$52^{\circ} 36^{\prime} 24^{\prime \prime} \mathrm{W}$ a $\left.53^{\circ} 07^{\prime} 43^{\prime \prime} \mathrm{W}\right)$ e General Carneiro $\left(26^{\circ} 20^{\prime} 35^{\prime \prime} \mathrm{S}\right.$ a $26^{\circ} 26^{\prime} 13^{\prime \prime} \mathrm{S}$ e $51^{\circ} 19^{\prime} 49^{\prime \prime} \mathrm{W}$ a $\left.51^{\circ} 25^{\prime} 29^{\prime \prime} \mathrm{W}\right)$, no Sul do estado do Paraná. Os plantios foram implementados, nos anos de 1987 e 1988 e anos de 1969 a 1987 , respectivamente.

Segundo a classificação de Köppen, o clima da região é do tipo $\mathrm{Cfb}$, subtropical mesotérmico, com temperatura média anual de $19,5^{\circ}$, precipitação média anual de 1.900 $\mathrm{mm}$ e com ocorrência de geadas (ALVARES et al., 2013). Os solos predominantes da região são classificados como Nitossolos e Neossolos (BHERING et al., 2007).

\section{Caracterização da área de estudo}

Para esse trabalho foram selecionadas e abatidas 30 árvores de Araucaria angustifolia entre os anos de 2003 e 2004. A seleção das árvores compreendeu os valores de diâmetros variando entre 14,3 a $33,4 \mathrm{~cm}$, dispostas em quatro classes de dap (diâmetro à altura do peito) com amplitude das classes de aproximadamente cinco centímetros. O número de árvores utilizados nesse estudo foi definido com base em estudos que apontam valores de 10 a 40 árvores foram satisfatórios para os ajustes de equações (SOARES et al., 2011, PELISSARI et al., 2011, SANQUETTA et al., 2015).

Foi mensurado o diâmetro à 1,3 metros de altura (dap) e após o corte foi mensurado o comprimento total que corresponde a altura total (ht) da árvore em pé, considerando uma altura de toco de $10 \mathrm{~cm}$. A quantificação da biomassa aérea verde foi realizada pelo método direto, separando cada árvore em três compartimentos para a pesagem: fuste com casca, acículas e galhos.

Em seguida foram selecionadas porções amostrais representativas da biomassa das acículas, dos galhos e do fuste sem casca, com massa variando de 300-500 g por compartimento, com objetivo de determinar a biomassa seca.

O material verde foi levado para estufa de renovação de ar à $70{ }^{\circ} \mathrm{C}$ até atingir peso constante. As porções amostrais secas foram pesadas em balança eletrônica de precisão de um grama e os percentuais de biomassa de cada compartimento foram calculados com base na 
equação abaixo:

$M s(\%)=\frac{P s}{P v} * 100$

Em que:

Ms (\%) = percentual de matéria seca;

$\mathrm{Ps}=$ peso seco total do compartimento (g); e

$\mathrm{Pv}=$ peso verde total do compartimento $(\mathrm{g})$.

A densidade básica da madeira $(\rho)$ foi determina a partir dos discos do lenho do fuste (sem casca), utilizando do método da balança hidrostática, conhecido, também, como método gravimétrico. Após a determinação da densidade básica da amostra, foi possível calcular a biomassa seca total de cada compartimento para cada indivíduo de $A$. angustifolia.

\section{Análises de modelagem da biomassa}

Foi realizada a análise de correlação de Pearson a 95\% de probabilidade visando melhor entender a relação entre as variáveis mensuradas. As variáveis utilizadas foram dap, ht, $\rho$, biomassa seca do fuste, biomassa seca da copa, que considera a soma da biomassa seca dos galhos e das acículas e a biomassa seca aérea total.

Foram testados 10 modelos alométricos lineares para cada compartimento. Para os modelos, os valores de biomassa seca dos diferentes compartimentos foram usados como variável dependente e os valores de dap, ht e $\rho$ como variáveis independentes (Tabela 1 ).

Tabela 1. Modelos alométricos utilizados para estimativa da biomassa em araucária, oriundos de povoamentos dos municípios de Quedas do Iguaçu e General Carneiro, Paraná

\begin{tabular}{|c|c|}
\hline \multicolumn{2}{|r|}{ Modelos } \\
\hline 1 & $Y=\beta_{0}+\beta_{1} \cdot$ dap $+\varepsilon$ \\
\hline 2 & $Y=\beta_{0}+\beta_{1} \cdot h t+\varepsilon$ \\
\hline 3 & $Y=\beta_{0}+\beta_{1} \rho+\varepsilon$ \\
\hline 4 & $Y=\beta_{0}+\beta_{1} \cdot$ dap $+\beta_{2} \cdot h t+\varepsilon$ \\
\hline 5 & $Y=\beta_{0}+\beta_{1} \cdot$ dap $+\beta_{2} \rho+\varepsilon$ \\
\hline 6 & $Y=\beta_{0}+\beta_{1} \cdot$ dap $^{2} h t+\beta_{2} \rho+\varepsilon$ \\
\hline 7 & $Y=\beta_{0}+\beta_{1} \cdot \operatorname{dap}+\beta_{2} h t+\beta_{3} \rho+\varepsilon$ \\
\hline 8 & $\log Y=\beta_{0}+\beta_{1} \cdot \log ($ dap $)+\varepsilon$ \\
\hline 9 & $\log Y=\beta_{0}+\beta_{1} \cdot \log ($ dap $)+\beta_{2} \cdot \log (h t)+\varepsilon$ \\
\hline 10 & $\log Y=\beta_{0}+\beta_{1} . \log ($ dap $)+\beta_{2} \log (h t)+\beta_{3} \log (\rho)+\varepsilon$ \\
\hline
\end{tabular}

A avaliação dos desempenhos dos modelos foi feita através dos critérios: coeficiente de determinação de
Schlaegel $\left(R^{2}{ }_{\mathrm{aj}}\right)$, erro padrão da estimativa em percentual $\left(S_{y x} \%\right)$, critério de informação de Akaike (AIC) e análise gráfica dos resíduos para os melhores modelos. A homogeneidade da variância foi verificada pelo teste de Breusch-Pagan, a normalidade dos resíduos foi verificada através do teste de Shapiro-Wilk e a independência dos resíduos foi verificada através do teste de Durbin-Watson. Todos os testes tiveram probabilidade de $95 \%$.

Após a verificação das condicionantes da regressão, no modelo escolhido foi aplicada a estatística da distância Cook para a detecção de valores outliers. Os indivíduos, que apresentam a distância Cook duas vezes maior do que a média de todas as distâncias, foram retirados. Após isso, reajustou-se o modelo de regressão.

Adicionalmente foi avaliado o ajuste simultâneo da biomassa seca por um sistema de equações, permitindo compatibilizar as estimativas. Tal procedimento visa que o resultado do somatório das equações de cada compartimento se assemelhe ao da equação para biomassa seca aérea total, garantindo a aditividade dos componentes de biomassa da árvore e melhora do ajuste. Para todos os procedimentos estatísticos foi utilizado o software R (R CORE TEAM, 2017).

\section{RESULTADOS E DISCUSSÃO}

Os valores médios de dap, ht e $\rho$ foram de $24,7 \mathrm{~cm}$, $16,7 \mathrm{~m}$ e $394,6 \mathrm{~kg} \cdot \mathrm{m}^{-3}$, onde a variável dependente que mais apresentou variação foi o dap, com coeficiente de variação de $22 \%$. O coeficiente de variação do dap, ht e $\rho$ foram inferiores ao da biomassa em qualquer compartimento para os indivíduos de $A$. angustifolia (Tabela 2).

Tabela 2. Estatística descritiva para as variáveis independentes e dependentes no plantio de araucária oriundos dos municípios de Quedas do Iguaçu e General Carneiro, Paraná

\begin{tabular}{cccccc}
\hline \multirow{2}{*}{ Variáveis } & \multicolumn{5}{c}{ Estatística descritiva } \\
\cline { 2 - 6 } & Média & Des. & Máx. & Mín. & $\mathrm{n}$ \\
\hline dap $(\mathrm{cm})$ & 24,7 & 5,6 & 33,4 & 14,3 & 30 \\
altura $(\mathrm{m})$ & 16,7 & 2,1 & 19,9 & 12,7 & 30 \\
$\rho\left(\mathrm{kg} \cdot \mathrm{m}^{3}\right)$ & 394,6 & 65,0 & 491,9 & 268,5 & 30 \\
B. copa $(\mathrm{kg})$ & 43,5 & 28,4 & 125,8 & 9,4 & 30 \\
B. fuste $(\mathrm{kg})$ & 179,0 & 101,5 & 402,4 & 45,4 & 30 \\
B. aérea $(\mathrm{kg})$ & 222,9 & 120,1 & 473,0 & 54,8 & 30 \\
\hline B. = Biomassa; Des. = Desvio; Máx. = maior valor; Mín. = menor valor; e \\
n = número de observações.
\end{tabular}

A biomassa do fuste representou $80,31 \%$ da biomassa 
aérea, enquanto a biomassa da copa apresentou 19,69\%, indicando que a maior parte da biomassa aérea está localizada no fuste das árvores. Os valores médios para a biomassa da copa e do fuste foram de 43,5 e 179,0 kg, respectivamente, onde a biomassa da copa apresentou a maior variação nos dados com coeficiente de variação de aproximadamente $65 \%$, enquanto que para a biomassa do fuste o coeficiente de variação foi de $56 \%$ (Tabela 2).

O comportamento da distribuição da biomassa nos diferentes compartimentos das plantas descrito nesse trabalho, está de acordo com o que preconiza a literatura, com acumulo maior da biomassa no fuste, galhos e, por último, nas folhas (SOCHER et al., 2008; SCHIKOWSKI et al., 2013).

Por meio da análise de correlação linear de Pearson foi possível entender o relacionamento das diferentes variáveis mensuradas nos indivíduos de $A$. angustifolia. Observa-se que as biomassas da copa, fuste e aérea apresentaram correlação significativa a $1 \%$ com o dap e ht (Tabela 3).

Tabela 3. Correlação linear de Pearson para as variáveis utilizadas nos modelos alométricos para plantio araucária oriundo dos municípios de Quedas do Iguaçu e General Carneiro, Paraná

\begin{tabular}{ccccccc}
\hline \multirow{2}{*}{ Correlação } & \multirow{2}{*}{ dap } & \multirow{2}{*}{ ht } & $\boldsymbol{\rho}$ & \multicolumn{3}{c}{ Biomassa } \\
\cline { 6 - 8 } & & & & copa & fuste & aérea \\
\hline dap & 1 & $0,85^{* *}$ & 0,11 & $0,60^{* *}$ & $0,92^{* *}$ & $0,92^{* *}$ \\
ht & - & 1 & 0,15 & $0,42^{* *}$ & $0,84^{* *}$ & $0,81^{* *}$ \\
$\rho$ & - & - & 1 & 0,20 & $0,44^{*}$ & $0,42^{*}$ \\
B. copa & - & - & - & 1 & $0,57^{* *}$ & $0,72^{* *}$ \\
B. fuste & - & - & - & - & 1 & $0,98^{* *}$ \\
B. aérea & - & - & - & - & - & 1 \\
\hline$*$
\end{tabular}

= significativo a $5 \%{ }^{* *}=$ significativo a $1 \%$.

Apesar de ambas serem significativas, o dap teve correlações mais fortes com a biomassa, principalmente do fuste, do que a variável ht, indicando que será uma variável essencial para estimar os valores de biomassa de A. angustifolia. Além disso, a resposta da biomassa do fuste às variáveis dendrológicas (dap e ht) é um fato conhecido e descrito em diversos trabalhos (ABOAL et al., 2005, VIEILLEDENT et al., 2012).

A densidade básica da madeira ( $\rho$ ) apresentou correlação significativa (nível de $5 \%$ de probabilidade) apenas com a biomassa do fuste e a biomassa aérea. Contudo as correlações não foram tão significativas quanto aquelas obtidas entre a biomassa e o dap. Segundo Basuki (2009) a variação da densidade básica da madeira, nos diferentes compartimentos da árvore, pode ser uma fonte de erro, ocasionando uma superestimação da biomassa nos modelos de árvores individuais.
Para estimativa da biomassa do fuste e aérea, com exceção dos modelos 2 e 3, todos tiveram valores de $\mathrm{R}^{2}$ maiores do que 0,8 e $\mathrm{S}_{y x} \%$ menores do que $25 \%$. Os melhores modelos foram os não logaritmizados e logaritmizados com dap, ht e $\rho$ como variáveis independentes, que apresentaram os menores valores de AIC, $S_{y x} \%$ e o maiores $R^{2}{ }_{\text {aj }}$ (Tabela 4), indicando que o dap, ht e $\rho$, quando utilizados conjuntamente nas equações, predizem a biomassa aérea e do fuste de maneira precisa.

Os ajustes dos modelos para estimativa da biomassa da copa não apresentaram estatísticas de seleção significativas como para a biomassa do fuste, visto que as variáveis independentes não apresentaram correlações fortes para esse compartimento. A biomassa de copa teve seus $\mathrm{R}^{2}$ aj variando de 0,01 a 0,35 e $\mathrm{S}_{\mathrm{yx}} \%$ entre 52 a $69 \%$. Os melhores modelos nesse caso foram os modelos $1,4,7$ e 8 , que apresentaram os maiores $\mathrm{R}^{2}{ }_{\mathrm{aj}}$, menores $\mathrm{S}_{\mathrm{yx}} \%$ e o menores AIC, sendo que esses modelos poderão ser usados, separadamente, no ajuste simultâneo dos modelos alométricos.

Para as condicionantes da regressão em relação a biomassa da copa, o teste de homogeneidade da variância de Breusch-Pagan preconizou que os resíduos foram heterogêneos nos modelos 1 e 2 . O teste de normalidade apontou que os resíduos apresentaram distribuição normal para todos os modelos. $O$ teste de independência dos resíduos de Durbin-Watson indicou que, para os modelos 5, 6, 7 e 8, os resíduos apresentaram autocorrelação (Tabela 5). Portanto, para a estimativa da biomassa de copa, os modelos que aderiram as condicionantes da regressão foram os modelos 3, 9 e 10. Dentre eles, o maior $\mathrm{R}^{2}$ aj e o menor $\mathrm{S}_{\mathrm{yx}} \%$ foi obtido no modelo 9, devendo esse ser usado preferencialmente no ajuste simultâneo.

Na biomassa do fuste, os modelos 2 e 3 apresentaram resíduos heterogêneos. O teste de normalidade dos resíduos indicou que o modelo 7 apresentou resíduos com distribuição não normal e o teste de independência dos resíduos preconizou que existe autocorrelação entre os resíduos dos modelos 1, 3, 8 e 9. Diante disso a utilização desses modelos para predição da biomassa do fuste não deve ser recomendada (Tabela 5).

Para a estimativa da biomassa do fuste é essencial que os resultados dos ajustes dos modelos de regressão linear sejam confiáveis, portanto, devem ser priorizados os modelos 4, 5, 6 e 10, que aderiram aos testes de condicionantes para a regressão. Pelo maior valor do $\mathrm{R}^{2}{ }_{\mathrm{aj}}$ $(0,99)$ e o menor $S_{y x} \%(0,21)$, dentre os quatro modelos citados, deve ser escolhido o modelo 10 para o ajuste simultâneo. 
Tabela 4. Coeficientes e critérios para a seleção dos modelos alométricos para estimativa da biomassa de diferentes compartimentos em plantio de araucária, oriundos de povoamentos florestais dos municípios de Quedas do Iguaçu e General Carneiro, Paraná

\begin{tabular}{|c|c|c|c|c|c|c|c|c|}
\hline & Modelos & $\beta_{0}$ & $\beta_{1}$ & $\boldsymbol{\beta}_{2}$ & $\boldsymbol{\beta}_{3}$ & $\mathbf{R}^{2}$ aj. & AIC & $\mathrm{S}_{\mathrm{yx}} \%$ \\
\hline \multirow{10}{*}{ 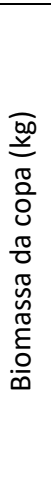 } & 1 & $-31,15$ & 3,04 & - & - & 0,34 & 277,3 & 52,47 \\
\hline & 2 & $-54,45$ & 5,89 & - & - & 0,16 & 284,7 & 59,36 \\
\hline & 3 & 9,33 & 0,09 & - & - & 0,01 & 289,6 & 64,40 \\
\hline & 4 & 8,33 & 4,44 & $-4,43$ & - & 0,35 & 278,0 & 52,26 \\
\hline & 5 & $-53,04$ & 2,97 & 0,06 & - & 0,34 & 278,4 & 52,65 \\
\hline & 6 & $-7,22$ & 0,003 & 0,05 & - & 0,29 & 280,5 & 54,48 \\
\hline & 7 & $-12,68$ & 4,50 & $-4,90$ & 0,07 & 0,35 & 278,7 & 52,17 \\
\hline & 8 & $-0,96$ & 1,82 & - & - & 0,31 & 0,1 & 52,57 \\
\hline & 9 & 0,21 & 2,73 & $-1,98$ & - & 0,32 & 0,3 & 52,18 \\
\hline & 10 & $-0,93$ & 2,81 & $-2,21$ & 0,50 & 0,26 & 1,4 & 54,23 \\
\hline \multirow{10}{*}{ 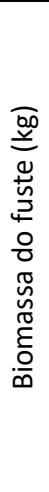 } & 1 & $-232,36$ & 16,66 & - & - & 0,85 & 310,2 & 22,28 \\
\hline & 2 & $-505,45$ & 40,98 & - & - & 0,69 & 331,3 & 31,70 \\
\hline & 3 & $-94,34$ & 0,69 & - & - & 0,17 & 360,7 & 51,74 \\
\hline & 4 & $-307,31$ & 14,01 & 8,41 & - & 0,85 & 310,6 & 22,09 \\
\hline & 5 & $-430,36$ & 15,98 & 0,54 & - & 0,97 & 263,3 & 10,05 \\
\hline & 6 & $-0,02$ & 0,02 & 0,48 & - & 0,98 & 248,1 & 7,80 \\
\hline & 7 & $-469,56$ & 14,49 & 4,76 & 0,53 & 0,97 & 262,7 & 9,79 \\
\hline & 8 & $-1,24$ & 2,47 & - & - & 0,87 & $-59,44$ & 20,27 \\
\hline & 9 & $-2,09$ & 1,82 & 1,43 & - & 0,87 & $-65,14$ & 20,13 \\
\hline & 10 & $-4,36$ & 1,98 & 0,98 & 1,00 & 0,99 & $-322,82$ & 0,21 \\
\hline \multirow{10}{*}{ 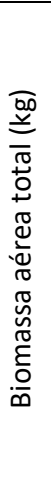 } & 1 & $-263,52$ & 19,70 & - & - & 0,85 & 320,3 & 21,17 \\
\hline & 2 & $-559,89$ & 46,87 & - & - & 0,64 & 345,6 & 32,28 \\
\hline & 3 & $-85,01$ & 0,78 & - & - & 0,15 & 371,5 & 49,68 \\
\hline & 4 & $-298,97$ & 18,45 & 3,97 & - & 0,84 & 322,1 & 21,47 \\
\hline & 5 & $-483,40$ & 18,95 & 0,60 & - & 0,95 & 285,4 & 11,65 \\
\hline & 6 & $-0,02$ & 0,02 & 0,53 & - & 0,95 & 289,5 & 12,48 \\
\hline & 7 & $-482,23$ & 18,99 & $-0,14$ & 0,60 & 0,95 & 287,4 & 11,87 \\
\hline & 8 & $-1,01$ & 2,38 & - & - & 0,86 & $-62,6$ & 19,63 \\
\hline & 9 & $-1,57$ & 1,95 & 0,95 & - & 0,85 & $-64,1$ & 20,25 \\
\hline & 10 & $-3,54$ & 2,09 & 0,56 & 0,87 & 0,96 & $-101,2$ & 10,27 \\
\hline
\end{tabular}

Tabela 5. Valores dos testes de homogeneidade, normalidade e independência dos resíduos para os modelos alométricos ajustados

\begin{tabular}{|c|c|c|c|c|c|c|c|c|c|c|c|}
\hline \multirow{2}{*}{ Variável } & \multirow{2}{*}{ Teste } & \multicolumn{10}{|c|}{ Modelo } \\
\hline & & 1 & 2 & 3 & 4 & 5 & 6 & 7 & 8 & 9 & 10 \\
\hline \multirow{3}{*}{ B. copa (kg) } & $\mathrm{BP}$ & $4,74^{*}$ & $5,67^{*}$ & 3,20 & $8,71^{*}$ & 3,00 & 4,53 & 3,11 & 0,02 & 1,71 & 3,59 \\
\hline & SW & 0,98 & 0,94 & 0,96 & 0,97 & 0,93 & 0,96 & 0,93 & 0,97 & 0,95 & 0,94 \\
\hline & DW & 1,44 & 2,58 & 1,40 & 1,58 & $2,87 * *$ & $2,77^{*}$ & $2,86^{*}$ & $1,01 * *$ & 1,37 & 1,98 \\
\hline \multirow{3}{*}{ B. fuste (kg) } & $\mathrm{BP}$ & 3,74 & $7,66^{* *}$ & $4,52 *$ & 5,26 & 0,31 & 1,05 & 1,39 & 0,23 & 0,87 & 7,46 \\
\hline & SW & 0,97 & 0,98 & 0,95 & 0,97 & 0,93 & 0,96 & $0,92^{*}$ & 0,95 & 0,95 & 0,97 \\
\hline & DW & $1,21 *$ & 2,33 & $1,19 *$ & 1,46 & 2,13 & 2,27 & 2,33 & $0,90 * *$ & $1,31 *$ & 1,45 \\
\hline \multirow{3}{*}{ B. aérea (kg) } & BP & $4,73^{*}$ & $5,67^{*}$ & 3,20 & $8,71^{*}$ & 3,00 & 4,53 & 3,11 & 0,02 & 1,71 & 3,59 \\
\hline & SW & 0,98 & 0,94 & 0,96 & 0,97 & 0,94 & 0,96 & 0,93 & 0,97 & 0,95 & 0,94 \\
\hline & DW & 1,44 & 2,58 & 1,40 & 1,58 & $2,87^{*}$ & $2,77^{*}$ & $2,86^{*}$ & $1,01 * *$ & 1,37 & 1,98 \\
\hline
\end{tabular}


A estimativa da biomassa aérea total seguiu comportamento parecido com a biomassa da copa, onde os únicos modelos que passaram pelas condicionantes de homogeneidade, normalidade e independência dos resíduos foram os modelos 3,9 e 10, tendo sido eleito o modelo 10 por apresentar as melhores estatísticas de ajuste.

O teste da distância Cook foi aplicado nos modelos 9 para a estimativa biomassa de copa e no modelo 10, para a estimativa da biomassa do fuste e aérea, visando retirar os valores influentes para aumentar a precisão das estimativas nos ajustes. 0 teste indicou que dentre as 30 árvores usadas no ajuste dos modelos, três árvores deveriam ser retiradas.

As equações 9, para a biomassa de copa, e 10, para a biomassa do fuste e aérea, foram reajustadas. Assim obtive-se a diminuição dos valores de $\mathrm{S}_{\mathrm{yx}} \%$ para todas as estimativas de biomassa, sendo mais evidente para a biomassa de copa (Tabela 6). Isso indica que os modelos ficaram mais precisos, evidenciando a importância de remover nesse caso, os valores influentes, conforme relatado nos trabalhos de Zhu, Ibrahim e Cho (2012) e Sartori et al. (2012).

Tabela 6. Coeficientes e critérios para a seleção dos modelos, após retirada dos valores influentes de acordo com o teste de distância Cook

\begin{tabular}{lccccccc}
\hline \multicolumn{2}{c}{ Modelos } & $\boldsymbol{\beta}_{\mathbf{0}}$ & $\boldsymbol{\beta}_{\mathbf{1}}$ & $\boldsymbol{\beta}_{\mathbf{2}}$ & $\boldsymbol{\beta}_{\mathbf{3}}$ & $\mathbf{R}_{\text {aj. }}$ & $\boldsymbol{S}_{\mathbf{y x}} \boldsymbol{\%}$ \\
\hline B. copa & 9 & 0,2079 & 2,7252 & $-1,9814$ & - & 0,40 & 44,59 \\
B.fuste & 10 & $-4,3663$ & 1,9854 & 0,9916 & 1,0006 & 0,99 & 0,14 \\
B.aérea & 10 & $-3,7160$ & 2,1541 & 0,2195 & 1,0626 & 0,98 & 7,34 \\
\hline
\end{tabular}

B. = biomassa $(\mathrm{kg})$

Para o ajuste simultâneo foram consideradas as melhores equações na estimativa da biomassa de cada compartimento para estimativa da biomassa aérea. Observa-se que o uso do ajuste simultâneo não ocasionou melhoras nas estatísticas de seleção dos modelos, visto o $\mathrm{R}^{2}{ }_{\mathrm{aj}} \mathrm{e} \mathrm{S}_{\mathrm{yx}} \%$ para a biomassa da copa manteve-se semelhante ao ajuste individual e o mesmo pode ser observado para os demais compartimentos (Tabela 7). A baixa previsibilidade da biomassa para copa das árvores pode estar associada a densidade de plantio, idade, sítio, geometria da copa entre outros fatores que influenciaram na variabilidade de crescimento entre os indivíduos.

Behling et al. (2012) e Coutinho et al. (2017) também relataram que o ajuste simultâneo não melhorou o desempenho das equações, contudo os autores orientaram que a vantagem de se utilizar o ajuste simultâneo está no fato de evitar distorções no somatório da biomassa obtida por meio das equações individuais de cada compartimento, que pode acontecer no ajuste independente.

Tabela 7. Resultados dos ajustes das equações simultâneas para a biomassa de cada compartimento e para a biomassa aérea

\begin{tabular}{|c|c|c|c|c|c|c|}
\hline Modelos & $\beta_{0}$ & $\beta_{1}$ & $\beta_{2}$ & $\beta_{3}$ & $\mathbf{R}^{2}{ }_{\text {aj }}$ & $S_{y x} \%$ \\
\hline \multicolumn{7}{|c|}{$\log (B . c o p a)=\beta_{0}+\beta_{1} \log ($ dap $)+\beta_{2} \log (h t)$} \\
\hline & 0,1887 & 2,6333 & $-1,8468$ & - & 0,41 & 44,5 \\
\hline \multicolumn{7}{|c|}{$\log ($ B.fuste $)=\beta_{0}+\beta_{1} \log ($ dap $)+\beta_{2} \log (h t)+\beta_{3} \log (\rho)$} \\
\hline & $-4,3702$ & 1,9859 & 0,9891 & 1,003 & 0,99 & 0,13 \\
\hline \multicolumn{7}{|c|}{$\log ($ B.aerea $)=\log ($ B.copa $)+\log$ (B.fuste) } \\
\hline & - & - & - & - & 0,96 & 10,4 \\
\hline
\end{tabular}

$\mathrm{Na}$ análise gráfica dos resíduos, observa-se que não foram encontradas diferenças na estimativa da biomassa da copa e do fuste, considerando o ajuste individual dos modelos 9 e 10 com o ajuste simultâneo, onde para a biomassa da copa, os modelos tenderam a superestimar os resultados (Figura 1).

$\mathrm{Na}$ biomassa do fuste, os resíduos foram menores do que $2 \%$, indicando o modelo foi altamente preciso e sem tendência na estimativa da biomassa, visto o elevado valor de correlação da biomassa fuste com as variáveis dendrométricas, principalmente o dap. Para estimativa da biomassa aérea, o ajuste simultâneo foi ligeiramente pior do que o ajuste individual do modelo 10, apresentando resíduos que chegaram a $30 \%$.

\section{CONCLUSÕES}

A biomassa da copa não apresenta forte relação com as variáveis dendrométricas como dap, ht e $\rho$, preconizando que a estimativa da biomassa considerando essas variáveis não é satisfatória. Assim indica-se a busca por variáveis que representam melhor a copa da árvore para o ajuste dos modelos alométricos.

A biomassa do fuste apresenta forte relação com o dap e ht, além da vantagem de serem variáveis de fácil mensuração.

Os modelos alométricos propostos no trabalho resultaram em valores satisfatórios dados os critérios de seleção para a estimativa da biomassa do fuste e biomassa aérea total.

O ajuste simultâneo garante a aditividade dos componentes, melhorando a estimativa da biomassa total, podendo ser usado no ajuste de modelos alométricos, contudo não alterar os valores dos coeficientes dos modelos de regressão utilizados. 
Biomassa Copa - Modelo 9

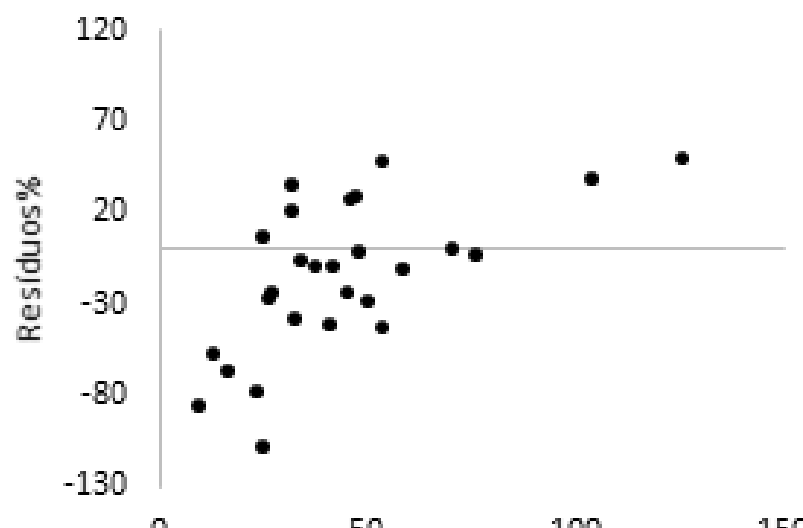

Biomassa copa observada (kg)

Biomassa fuste - Modelo 10

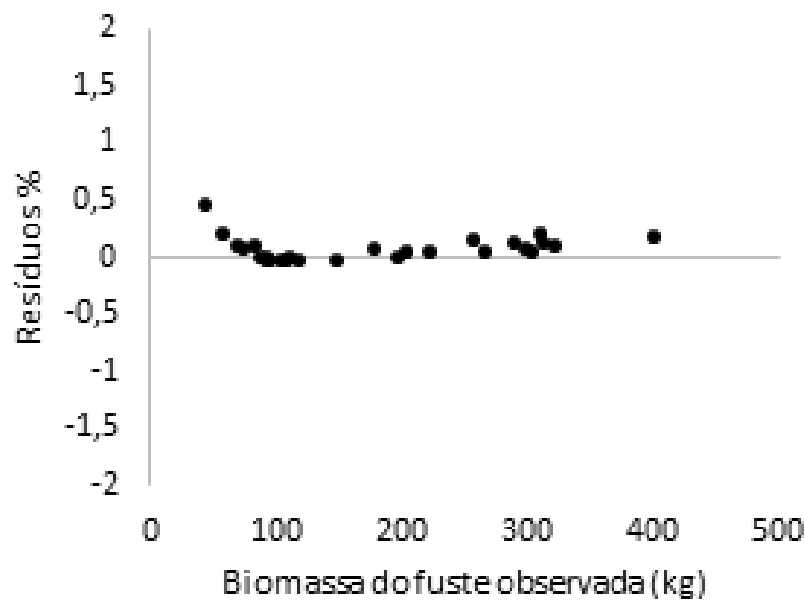

Biomassa aérea - Modelo 10

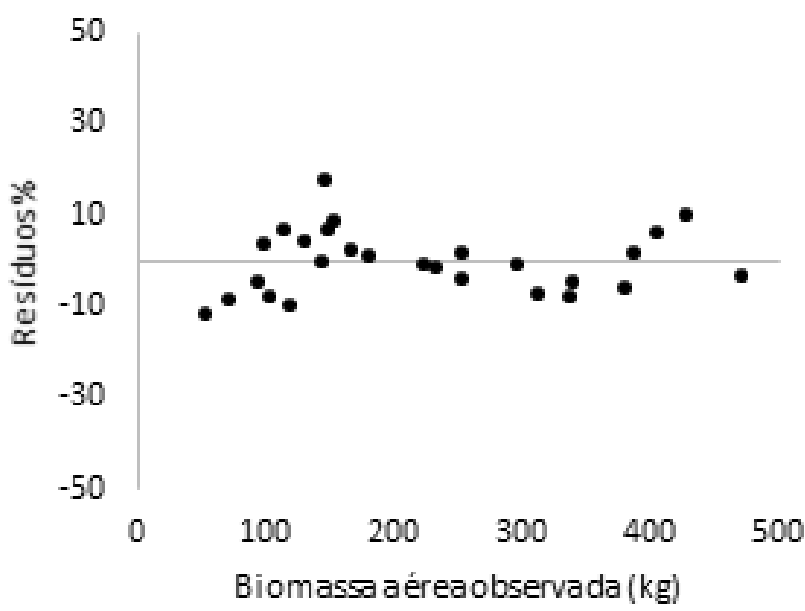

Biomassa copa - Ajuste simultâneo

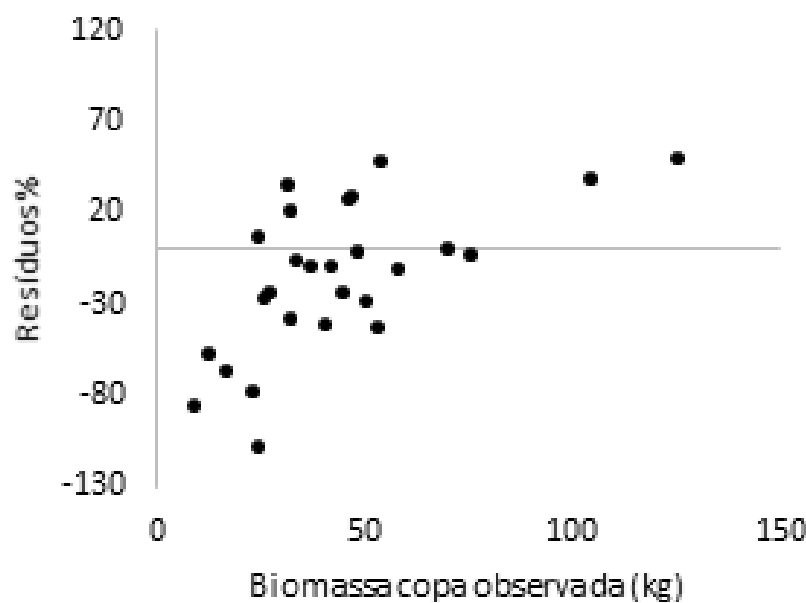

Biomassa fuste - Ajuste simultâneo

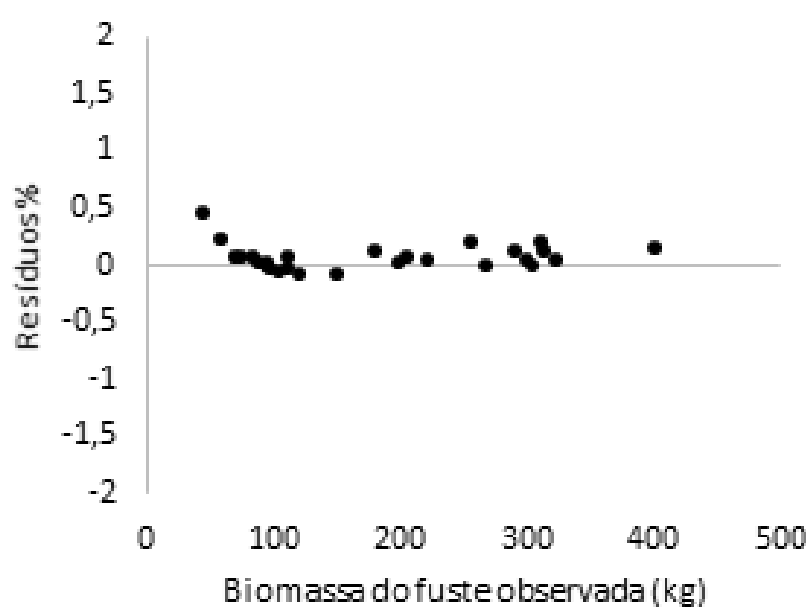

Biomassa aérea - Ajuste simultâneo

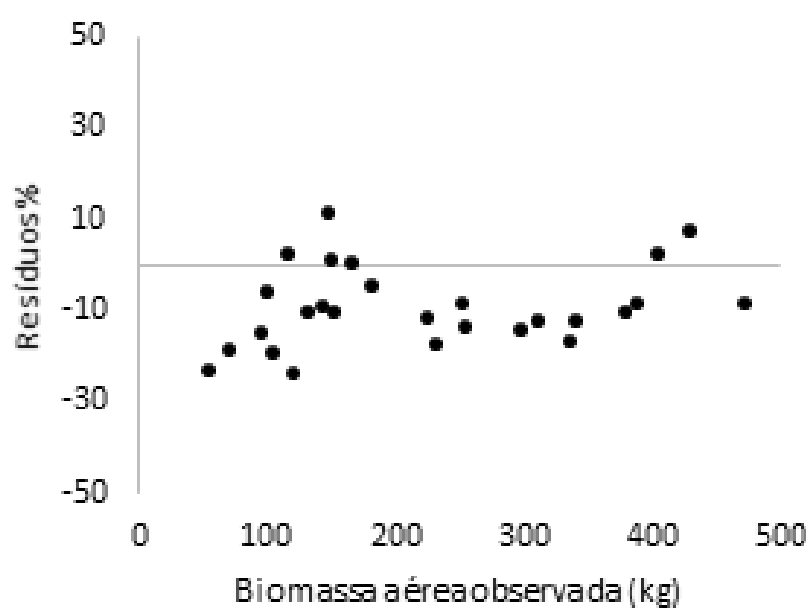

Figura 1. Distribuição de resíduos para a estimativa de biomassa de A. angustifolia oriundas de povoamentos florestais dos municípios de Quedas do Iguaçu e General Carneiro, Paraná, considerando a modelagem individual e o ajuste simultâneo. 


\section{REFERÊNCIAS}

ABOAL, J.R et al. Allometric relationships of different tree species and stand above ground biomass in the Gomera laurel forest (Canary Islands). Flora, n.200, p.264-274, 2005.

ALVARES, C.A. et al. Köppen's climate classification map for Brasil. Meteorologische Zeitschrift, v.22, n.6, p.711-728, 2013.

ANTÓNIO, N. et al. Effect of tree, stand, and site variables on the allometry of Eucalyptus globulus tree biomass. Canadian Journal of Forest Research, v.37, p.895-906, 2007.

BASUKI, T.M. et al. Allometric equations for estimating the above-ground biomass in tropical lowland Dipterocarp forests. Forest Ecology and Management, v.257, n.8, p.1684-1694, 2009.

BEHLING, A. et al. Equações simultâneas para estimativa da biomassa em plantios comerciais de acácia-negra. Enciclopédia Biosfera, v.8, n.15, p.853-860, 2012.

BHERING, S.B. et al. Mapa de solos do estado do Paraná. Rio de Janeiro: Embrapa Solos, 2007. (Documentos 96)

CARVALHO, J.P.; PARRESOL, B.R. Additivity in tree biomass components of Pyrenean oak (Quercus pyrenaica Willd.). Forest Ecology and Management, v. 179, p. 269-276, 2003.

COUTINHO, V.M. et al. Equações tradicionais e simultâneas para biomassa de compartimentos aéreos de Cryptomeria japonica (L.F.) D. Don. Revista Brasileira de Biometria, v.35, n.1, p.58-75, 2017.

CUBAS, R. et al. Modelagem da biomassa da regeneração natural em plantio de Pinus. Pesquisa Florestal Brasileira, v.36, n.87, p.303-310, 2016.

IPCC. Climate Change 2014 Synthesis Report Summary Chapter for Policymakers. Intergovernmental Painel on Climate Change, 2014. Disponível em: https://doi.org/10.1017/СBO9781107 $\underline{415324}$

NICODEMO, M.L.F. et al. Allometric models for estimating aboveground biomass and biomass allocation of capixingui trees (Croton floribundus Spreng.) inana agrisilvicultural system. Revista Árvore, v. 40, n.2, p.279-288, 2016.

NICOLETTI, M.F. et al. Metodologia não destrutiva para a quantificação do volume e biomassa do fuste em remanescente florestal. Nativa, v.3, n.4, p.287-291, 2015.

PELISSARI, A.L. et al. Modelos volumétricos para Pinus tropicais, em povoamento homogêneo, no Estado de Rondônia. Pesquisa Florestal Brasileira, v.31, n.67, p.173-181, 2011.

$R$ CORE TEAM. R: A language and environment for statistical computing. Vienna: R Foundation for Statistical Computing, 2017. Disponível em: https://www.R-project.org

SANQUETTA, C.R. et al. Simultaneous estimation as alternative to independent modeling of tree biomass. Annals of Forest Science, v.72, p.1099-1112, 2015.

SANQUETTA, C.R. et al. Estimativa de carbono individual para Araucaria angustifolia. Pesquisa Agropecuária Tropical, v.44, n.1, p.1-8, 2014.

SANQUETTA, C.R. et al. A.A. Relações individuais de biomassa e conteúdo de carbono em plantações de Araucaria angustifolia e Pinus taeda no sul do estado do Paraná, Brasil. Revista Acadêmica: ciências agrárias e ambientais, v.1, n.3, p.33-40, 2003.

SARTORI, L.R. et al. Atributos polarimétricos de imagem de radar na inferência de parâmetros morfológicos de macrófitas. Boletim de Ciências Geodésicas, v. 18, n.1, p.138-153, 2012.

SCHIKOWSKI, A.B. et al. Modelagem do crescimento e da biomassa individual de Pinus. Pesquisa Florestal Brasileira, v.33, n.75, p.269-278, 2013.

SOARES, C.P.B. et al. Influence of section lenght on volume determination in Eucalyptus tress. Cerne, v.16, n.2, p.155-162, 2010.

SOCHER, L.G. et al. Biomassa aérea de uma Floresta Ombrófila Mista aluvial no município de Araucária (PR). Floresta, v.38, n.2, p.245-252, 2008.

SOUZA, A.L. et al. Estrutura fitossociológica, estoques de volume, biomassa, carbono e dióxido de carbono em floresta estacional semidecidual. Revista Árvore, v.36, n.1, p.169-179, 2012.

VIANA, H. et al. Predição da biomassa aérea da Pinus pinaster Aiton por um sistema de equações aditivas integrado no simulador Open Source ModisPinaster. Silva Lusitana, v.21, n. Especial, p.77-86, 2013.

VIEILLEDENT, G. et al. A universal approach to estimate biomass and carbon stock in tropical forests using generic allometric models. Ecological Applications, n.22, p.572-583, 2012.

WATZLAWICK, L.F. et al. Variação dos teores de carbono orgânico em espécies arbóreas da floresta ombrófila mista. Floresta e Ambiente, v.18, n.3, p.248-258, 2011.

ZHU, B.H. et al. Permutation and scaled Cook's distance. The Annals of Statistica, v.40, n.2, p.785-811, 2012 